\title{
Padrão alimentar de crianças de 6 a 24 meses em área rural do Maranhão*
}

\author{
Alimentary standard of children aged 6 to 24 months in rual area of Maranhão \\ Padrón alimentício de niños de 6 a 24 meses em area rural de Maranhão
}

\author{
Francisca Georgina Macedo de Sousa1', Thelma Leite de Araújo²
}

\begin{abstract}
RESUMO: Estudo exploratório transversal com o objetivo de identificar práticas alimentares infantis adotadas por famílias da Vila São Pedro, Paço do Lumiar - MA - Brasil. A população do estudo foi representada por crianças na faixa etária de 6 a 24 meses totalizando 118 crianças. Os dados subjetivos foram coletados em entrevistas individuais com as mães utilizando-se as variáveis do Elemento Estilo de Vida do Modelo Campo de Saúde de Lalonde. Na população estudada o aleitamento materno foi amplamente praticado, pois $89,8 \%$ das mães disseram ter amamentado seus filhos. No entanto, 97,4\% das crianças receberam chás, água e espessantes antes dos seis meses de vida e somente $2,6 \%$ das crianças foram mantidas em aleitamento materno exclusivo. As frutas não foram adequadamente introduzidas na dieta infantil. O mingau de cereal é, para $62,7 \%$ das crianças, o único alimento, caracterizando uma dieta monótona e pobre em nutrientes. Tais resultados podem orientar ações de monitoramento e reorientação às ações de suporte à alimentação infantil.
\end{abstract}

Descritores: Aleitamento materno; Alimentação mista; Alimentação artificial

ABSTRACT: The main goal of the transversal exploratory survey was to identify infantile alimentary practices adopted by families from the Village of São Pedro, in the municipality of Paço do Lumiar, Maranhão Northeastern of Brazil. The target population was represented by children aged 6 to 24 months, 118 altogether. The survey's data was collected through individual interviews with mothers. The survey found that in the target population, the maternal breastfeeding was widely practiced, therefore $, 89,8 \%$ of the mothers informed that they breastfed their children. However, $97,4 \%$ of children had received teas, water and thick aliments before the six months of living and $2,6 \%$ of the children were exclusively breastfed. The fruits not been introduced in the children's diet properly. Cereals soup is the only kind of food for $62,7 \%$ of the children, characterizing a monotonous and poor diet in nutrients. Such results can lead us to prioritize the monitoring and reorientation to the actions or support to the infantile feeding.

Keywords: Breast feeding; Mixed feeding; Bottle feeding

RESUMEN: Estudio exploratorio, transversal con el objetivo principal de identificar practicas alimentares infantiles adoptadas por famílias en la Villa São Pedro, Paço do Lumiar. La muestra del estudio fue representada por niños en edad entre 6 a 24 meses. Los datos subjetivos fuerón acopiados en entrevistas individuales con las madres. Los resultados mostraron que el amamantamiento materno fue ampliamente practicado, pues $89,8 \%$ de las madres dijerón ter amamantado sus hijos, mas $97,4 \%$ dos los niños recibierón te, líquidos y papas antes de los 6 meses de edad y solo 2,6\% de los niños fuerón mantenidos con leche materno exclusivo. Las frutas no fueron introducidas en la dieta infantil. Las papas de cereales son para $62,7 \%$ de los niños el único alimento, caracterizando una dieta monótona y pobre en nutrientes. Esos resultados pueden orientar acciones de monitoramento y reorientación a las acciones de soporte de alimentación infantil.

Descriptores: Lactancia materna; Alimentación mixta; Alimentación artificial

\section{INTRODUÇÃO}

Dentre os vários aspectos da saúde da criança, a alimentação, reveste-se de fundamental importância por oferecer tanto nos aspectos qualitativos quanto quantitativos, nutrientes que atendam às necessidades do cres- cimento e do desenvolvimento infantil. Cumpre assinalar, nesse particular, que o crescimento intenso e rápido da criança, principalmente nos primeiros dois anos de vida, deve ser acompanhado de alimentação adequada para atender às necessidades de macro e micronutrientes ${ }^{(1)}$. Nesse sentido, enfatizam-se as vantagens da

\footnotetext{
* Derivado da dissertação: Sousa FGM. Situação nutricional de ferro em crianças de seis a sessenta meses na perspectiva do Modelo Campo de Saúde [dissertação]. Fortaleza (CE); Programa de Pós-Graduação em Enfermagem/UFC; 2002.

1 Enfermeira. Mestre em Enfermagem. docente da UFMA, Doutoranda do Programa de Pós-Graduação em Enfermagem da Universidade Federal de Santa Catarina -UFSC. E - mail: fgeorginams@hotmail.com / franciscagms@nfr.ufsc.br

2 Enfermeira. Doutora em Enfermagem. Adjunto da Universidade Federal do Ceará/UFC. Pesquisadora CNPq.
} 
amamentação, pois garante, em muitos casos, a sobrevivência das crianças, e, em virtude das propriedades antiinfecciosas do leite materno as protege contra diferentes infecções desde os primeiros dias de $v_{i d a}{ }^{(2)}$.

Os profissionais de saúde e a família devem promover estratégias para assegurar adequada ingestão de alimentos nutritivos e energéticos. Neste sentido é necessário observar a quantidade, a qualidade, a harmonia e a adequação dos alimentos oferecidos às criança ${ }^{(3)}$. Práticas inadequadas de suplementação alimentar poderão ter impacto negativo na saúde da criança com repercussões na fase $\operatorname{adulta}^{(4)}$. No que diz respeito às repercussões decorrentes de inadequação alimentar destacam-se dois mecanismos: o primeiro, é o efeito cumulativo de alterações que embora se iniciem precocemente na vida, resultam em morbidade anos depois. O segundo, é a formação de hábitos alimentares que conduzem a práticas dietéticas indesejáveis, contribuindo para a deficiência de alguns nutrientes, principalmente dos micronutrientes $^{(5)}$.

Para o Ministério da Saúde, alimentação infantil adequada compreende a prática do aleitamento materno e a introdução, em tempo oportuno de alimentos apropriados que complementam o aleitamento materno ${ }^{(2)}$. A recomendação da Organização Mundial de Saúde apoiada pelo Ministério da Saúde é garantir o aleitamento materno exclusivo até os seis meses de vida e o aleitamento parcial até os dois anos de idade ${ }^{(6)}$. A partir do sexto mês, recomenda a introdução de alimentos complementares de forma lenta e gradual baseada nas evidências de que a introdução de alimentos complementares antes deste período não oferece vantagens e pode ser prejudicial à saúde da criança ${ }^{(2)}$. O argumento que sustenta a afirmação anterior diz respeito ao aumento da morbimortalidade infantil, especialmente em locais com precárias condições de higiene ${ }^{(7)}$. Nas últimas décadas, vários estudos têm descrito as modificações comportamentais e os mecanismos de controle da ingestão alimentar, principalmente na população infantil dada a vulnerabilidade deste grupo etário. Estudiosos afirmam que as práticas alimentares sofreram adaptações muitas vezes pouco saudáveis o que constitui desvantagens para a saúde ${ }^{(4)}$. Por tais afirmativas, considera-se importante investigar as práticas alimentares, questionando-se neste estudo, qual o padrão alimentar das crianças de 6 a 24 meses na Vila São Pedro, área rural maranhense.

\section{OBJETIVOS}

- identificar e descrever as práticas alimentares e o padrão alimentar de crianças de 6 a 24 meses, inscritas no Programa de Saúde da Família (PSF) de uma comunidade rural maranhense.

\section{MÉTODO}

Trata-se de um estudo exploratório, transversal inserido em uma proposta mais ampla que teve como finalidade investigar a situação nutricional de ferro na população de 6 a 60 meses de idade ${ }^{(8)}$.

Para este estudo foi feito um recorte, tendo como objeto as práticas e o padrão alimentar de crianças de 6 a 24 meses residentes em uma comunidade rural maranhense cujas famílias estavam cadastradas no PSF. A população do estudo foi identificada a partir das Fichas de Cadastro desse Programa totalizando 118 crianças na faixa etária de 6 a 24 meses. As informações sobre as práticas e padrão alimentar foram coletadas com as mães das crianças utilizando-se formulário estruturado baseado no Modelo Campo de Saúde ${ }^{(9)}$, selecionando-se para este estudo, as variáveis do Elemento Estilo de Vida (Anexo A). As variáveis para este elemento constituíram-se da história alimentar da criança, alimentos usados na dieta e freqüência da alimentação infantil. As mães foram entrevistadas individualmente em locais e horários previamente negociados, inclusive nos finais de semana. As informações maternas que diziam respeito a dados retrospectivos, foram analisadas com cuidado pelas pesquisadoras e, em algumas vezes, obrigou-as a retornar às mães para que dúvidas fossem esclarecidas.

Para caracterizar o padrão alimentar das crianças pesquisadas, utilizou-se a classificação adotada pelo Ministério da Saúde o qual define três categorias de aleitamento materno: a primeira, aleitamento materno exclusivo, quando a criança recebe somente leite materno diretamente da mama ou extraído, e nenhum outro líquido ou sólido, com exceção de gotas ou xaropes de vitaminas, minerais ou medicamentos. A segunda, aleitamento materno predominante, quando o lactente recebe, além do leite materno, água ou bebidas à base de água, como sucos de frutas e chás. Por último, aleitamento materno, quando a criança recebe leite materno, diretamente do seio ou extraído, independente de estar recebendo qualquer alimento ou líquido, incluindo leite nãohumano. Associa-se a esta classificação a alimentação artificial, quando o lactente recebe leite não-humano ${ }^{(2)}$.

Quanto aos aspectos éticos, o estudo foi aprovado pelo Comitê de Ética e Pesquisa do Hospital Universitário da Universidade Federal do Maranhão, em concordância com o que determina a Resolução n 196/96 do Conselho Nacional de Saúde ${ }^{(10)}$.

\section{RESULTADOS}

Os resultados a seguir apresentados reafirmam a heterogeneidade no padrão alimentar da população infantil, vivenciada também na prática clínica, sendo este um dos maiores desafios para os profissionais envolvidos no cuidado à criança. 
Tabela 1 - Crianças segundo faixa etária, aleitamento materno e complemento do leite, Vila São Pedro, Paço do Lumiar - MA, 2002.

\begin{tabular}{lcc}
\hline VARIÁVEIS & N & $\%$ \\
\hline Faixa Etária & & \\
$6-11$ meses & 25 & 21,2 \\
$12-18$ meses & 34 & 28,8 \\
$19-24$ meses & 59 & 50,0 \\
\hline Total & 118 & 100,0 \\
\hline Aleitamento Materno & & \\
Sim & 106 & 89,8 \\
Não & 12 & 10,2 \\
\hline Total & 118 & 100,0 \\
\hline Padrão de Aleitamento Materno & & \\
Aleitamento materno exclusivo & 03 & 2,6 \\
Aleitamento materno predominante & 71 & 60,1 \\
Aleitamento materno & 32 & 27,1 \\
Aleitamento artificial & 12 & 10,2 \\
\hline Total & $\mathbf{1 1 8}$ & $\mathbf{1 0 0 , 0}$ \\
\hline
\end{tabular}

O maior percentual de crianças do estudo encontrava-se na faixa etária de 19 a 24 meses com $50,0 \%$ do total, seguida por aquelas entre 12 e 18 meses $\operatorname{com} 28,8 \%$, enquanto que 21,2\% tinham entre 6 e 11 meses. Para caracterizar a prática do aleitamento materno e o padrão alimentar das crianças pesquisadas, recorreu-se a dados retrospectivos colhidos junto às mães, considerando que a população do estudo era constituída de crianças de 6 a 24 meses. $\mathrm{O}$ fato da mãe ter ou não amamentado, é uma ocorrência tão importante e marcante na vida da mulher que todas conseguem descrever esta experiência com riquezas de detalhes ou respaldadas em argumentos que justifiquem o insucesso da amamentação ${ }^{(7)}$. Nesse sentido, os resultados do estudo demonstram que $89,8 \%$ das mães amamentaram seus filhos em alguma época da fase de lactância, enquanto que $10,2 \%$ das crianças não receberam leite materno. Em regime de aleitamento materno exclusivo e predominante registrou-se respectivamente $2,6 \%$ e $60,1 \%$. Observa-se que houve prevalência do aleitamento materno predominante sobre as demais categorias, o que pode sugerir que as mães valorizam o leite materno, mas não ao ponto de tê-lo como único alimento para seus filhos no período de zero a seis meses de idade, como é preconizado.

A prevalência de aleitamento materno entre a população estudada foi de $27,1 \%$, enquanto que, $10,2 \%$ receberam como alimento o leite não-humano, incluídas, portanto, na categoria alimentação artificial.

Tabela 2 - Alimentos complementares e idade de introdução na alimentação das crianças estudadas, Vila São Pedro, Paço do Lumiar -MA, 2002.

\begin{tabular}{|c|c|c|c|c|c|c|c|c|}
\hline ALIMENTOS LÍQUIDOS & $\begin{array}{l}1 \text { sem a } \\
<1 \text { mês }\end{array}$ & 1 mês & $2-3 m$ & $4-5 m$ & $6-11 m$ & $12-18 m$ & $>18 \mathrm{~m}$ & Total \\
\hline Água/chás & $\begin{array}{c}68 \\
57,6 \%\end{array}$ & $\begin{array}{c}32 \\
27,2 \%\end{array}$ & $\begin{array}{c}15 \\
12,6 \%\end{array}$ & - & $\begin{array}{c}3 \\
2,6 \%\end{array}$ & - & - & $\begin{array}{c}118 \\
100 \%\end{array}$ \\
\hline Espessante & $\begin{array}{c}12 \\
10,2 \%\end{array}$ & $\begin{array}{c}37 \\
31,3 \%\end{array}$ & $\begin{array}{c}54 \\
45,7 \%\end{array}$ & $\begin{array}{c}12 \\
10,2 \%\end{array}$ & $\begin{array}{c}3 \\
2,6 \%\end{array}$ & - & - & $\begin{array}{c}118 \\
100 \%\end{array}$ \\
\hline Leite artificial & $\begin{array}{c}12 \\
10,1 \%\end{array}$ & $\begin{array}{c}22 \\
18,6 \%\end{array}$ & $\begin{array}{c}48 \\
40,7 \%\end{array}$ & $\begin{array}{c}12 \\
10,1 \%\end{array}$ & $\begin{array}{c}3 \\
2,6 \%\end{array}$ & - & - & $\begin{array}{c}97 \\
82,2 \%\end{array}$ \\
\hline Suco de frutas & - & - & $\begin{array}{c}2 \\
1,7 \%\end{array}$ & $\begin{array}{c}17 \\
14,4 \%\end{array}$ & $\begin{array}{c}12 \\
10,2\end{array}$ & - & - & $\begin{array}{c}31 \\
30,5 \%\end{array}$ \\
\hline Papinha de frutas & - & - & $\begin{array}{c}3 \\
2,5 \%\end{array}$ & $\begin{array}{c}3 \\
2,5 \%\end{array}$ & $\begin{array}{c}2 \\
1,7 \%\end{array}$ & - & - & $\begin{array}{c}8 \\
6,8 \%\end{array}$ \\
\hline Sopa & - & - & $\begin{array}{c}1 \\
0,8 \%\end{array}$ & $\begin{array}{c}4 \\
3,4 \%\end{array}$ & $\begin{array}{c}6 \\
5,1 \%\end{array}$ & - & - & $\begin{array}{c}11 \\
9,3 \%\end{array}$ \\
\hline Comida da família & - & - & - & - & $\begin{array}{c}4 \\
3,4 \%\end{array}$ & - & - & $\begin{array}{c}39 \\
33,0 \%\end{array}$ \\
\hline
\end{tabular}

Com exceção de três crianças $(2,6 \%)$, as demais receberam complementos do leite materno em idades precoces. A água e os chás, os espessantes e o leite artificial foram os itens da dieta com oferta iniciada antes do primeiro mês de vida com percentuais de $57,6 \%, 10,2 \%$ e $10,1 \%$, respectivamente. A introdução de espessantes à dieta infantil está relacionada ao aumento da saciedade particularmente à noite, permitindo a dupla mãe e filho um maior número de horas de sono ${ }^{(11)}$. Quanto à idade em que os complementos do leite materno foram introduzidos, chama atenção o fato de $57,6 \%$ das crianças ter recebido água ou chás ainda na primeira semana de vida. Segundo o Ministério da Saúde, isso ocorre principalmente em função de uma percepção por parte das 
mães e familiares de que o leite materno sozinho não é suficiente para sustentar o bebê, além do que, as mães entendem que as crianças têm sede e que essa sede não é saciada pelo leite materno ${ }^{(2)}$. Os chás são utilizados como remédios em casos de cólicas, dificuldade para dormir e para acalmar as crianças. No entanto, a complementação do leite materno com água ou chás nos primeiros seis meses é desnecessária e ainda concorre para diminuir o volume total do leite materno ${ }^{(12)}$.

Por outro lado a complementação das dietas infantis com frutas (suco /papinhas de frutas) e sopa foi baixa $(30,5 \%, 6,8 \%$ e $9,3 \%)$ respectivamente, sendo que as frutas são oferecidas com mais freqüência em forma de sucos $(30,5 \%)$. Apesar de indicada, a partir do sexto mês, um percentual baixo de crianças recebe a mesma comida da família $(33,0 \%)$. Fato que poderá excluir da dieta da criança alguns nutrientes que são normalmente consumidos na rotina dietética da família e, por outro lado, reforça a monotonia da dieta infantil.

Se comparado o número de crianças que receberam espessantes (118) e as que receberam leite artificial (97), há uma diferença de 21 crianças. Estas receberam mingau de farinha sem a adição do leite, pois as mães compreendem que o leite é prejudicial à saúde das crianças pequenas.

Ainda em relação à introdução de alimentos complementares os resultados apontam duas situações: aqueles alimentos introduzidos precocemente e, referidos na literatura científica, como desnecessários e aqueles introduzidos tardiamente ou não oferecidos, como é o caso das frutas, sopa e alimentação da família.

O Ministério da Saúde adverte que a alimentação da criança deve ser variada e, para facilitar a aceitação da criança ela deve ser exposta, na época oportuna, aos diferentes alimentos, ainda no primeiro ano de $\operatorname{vida}^{(2)}$.
Tabela 3 - Alegações maternas para o uso do leite artificial, Vila São Pedro, Paço do Lumiar - MA, 2002.

\begin{tabular}{lcc}
\hline ALEGAÇÕES MATERNAS & N & \% \\
\hline Leite fraco & 12 & 10,4 \\
Pouco leite nopeito & 19 & 16,5 \\
Leite fraco + pouco leite & 16 & 14,0 \\
Leite fraco + choro da criança & 53 & 46,1 \\
Choro da criança & 03 & 2,6 \\
Problemas de mama & 05 & 4,3 \\
Outros & 07 & 6,1 \\
\hline Total & $\mathbf{1 1 5}$ & $\mathbf{1 0 0 , 0}$ \\
\hline
\end{tabular}

Para as crianças classificadas em aleitamento materno predominante, aleitamento materno e alimentação artificial (115), as alegações mais freqüentes referidas pelas mães para a interrupção da amamentação exclusiva foi o leite fraco + choro da criança $(46,1 \%)$ e pouco leite no peito $(16,5 \%)$.

Estudo sobre o tema identificou que o leite fraco e o pouco leite são as principais alegações maternas para o desmame e a origem dessa informação vem do senso comum. São respostas socialmente aceitas para a incapacidade de a mãe verbalizar o insucesso da amamentação ${ }^{(13)}$.

Os problemas da mama, apesar de terem sido pouco referidos $(4,3 \%)$, podem deixar de existir caso as mães recebam no pré-natal e puerpério apoio e orientações que visem à prevenção dos mesmos, contribuindo dessa forma para que um maior número de crianças seja amamentado. O trabalho materno e a freqüência escolar foram outras alegações maternas para a introdução de outros alimentos na dieta da criança.

Tabela 4 - Alimentação atual e freqüência das refeições das crianças pesquisadas, Vila São Pedro, Paço do Lumiar MA, 2002.

\begin{tabular}{|c|c|c|c|c|}
\hline VARIÁVEIS & $6-11 \mathrm{~m}$ & $12-18 m$ & $19-24 m$ & Total \\
\hline \multicolumn{5}{|l|}{ Alimentação atual } \\
\hline Leite materno + mingaus & $8(32,0 \%)$ & $6(17,6 \%)$ & $2(3,4 \%)$ & $16(13,5 \%)$ \\
\hline Mingaus & $13(5,2 \%)$ & $14(41,2 \%)$ & $19(32,2 \%)$ & $46(39,0 \%)$ \\
\hline Mingaus + frutas & $2(8,0 \%)$ & $4(11,8 \%)$ & $6(10,2 \%)$ & $12(10,2 \%)$ \\
\hline Leite materno + sopa + frutas & $2(8,0 \%)$ & $3(8,8 \%)$ & - & $5(4,3 \%)$ \\
\hline Comida da família & - & $1(2,9 \%)$ & $22(37,3 \%)$ & $23(19,5 \%)$ \\
\hline Comida da família + frutas & - & $6(17,6 \%)$ & $10(16,9 \%)$ & $16(13,5 \%)$ \\
\hline Total & 25 & 34 & 26 & 118 \\
\hline \multicolumn{5}{|l|}{ Freqüência das refeições } \\
\hline 3 - 4 vezes & $4(16,0 \%)$ & $14(41,2 \%)$ & $19(32,2 \%)$ & $37(31,4 \%)$ \\
\hline $5-6$ vezes & $12(48,0 \%)$ & $17(50,0 \%)$ & $26(44,1 \%)$ & $55(46,6 \%)$ \\
\hline $6-7$ vezes & $9(3,6 \%)$ & $3(8,8 \%)$ & $14(23,7 \%)$ & $26(22,0 \%)$ \\
\hline Total & 24 & 34 & 59 & 118 \\
\hline
\end{tabular}


O leite materno associado a mingaus de cereais são os únicos alimentos para 13,5\% das crianças estudadas. O mingau de cereal aparece como opção alimentar para 74 crianças (leite materno + mingaus; mingaus; mingaus + frutas) e destas 46 crianças $(39,0 \%)$ o utilizam como única fonte alimentar caracterizando uma dieta monótona e de baixo teor protéico. O mais importante em uma dieta, do ponto de vista, nutricional, é a sua composição ${ }^{(14)}$ sendo recomendado que esta seja bastante variada, com o objetivo de diminuir problemas de saúde e estimular a adoção de práticas alimentares mais saudáveis ${ }^{(15)}$.

O Ministério da Saúde ${ }^{(2)}$ preconiza que o aleitamento materno se prolongue até os dois anos de idade, ao passo de que neste estudo, apenas $17,8 \%$ das crianças continuam sendo amamentadas até os 24 meses (correspondem as crianças que tem como opção dietética o leite materno + mingaus, leite materno + sopa + frutas).

No que diz respeito à freqüência com que as dietas são oferecidas, $31,4 \%$ das crianças recebem entre três e quatro, $46,6 \%$ entre cinco e seis e $22,0 \%$ seis a sete refeições diárias. A conduta apropriada resume-se em oferecer, a partir dos seis meses, seis refeições nas 24 horas, sendo duas refeições completas (com adição de carnes, vegetais e legumes) e as demais devem incluir sucos e frutas amassadas, mingaus de cereais e manter o aleitamento materno ${ }^{(16)}$.

\section{CONCLUSÃO}

Os resultados do estudo permitiram conhecer o padrão alimentar das crianças pesquisadas, classificando-o como inadequado, monótono, deficiente em micronutrientes representado, na maioria das vezes, pelos mingaus de cereais e pela baixa freqüência das dietas.

Confrontando o perfil de introdução dos alimentos nas crianças pesquisadas e o que é preconizado pelo Ministério da Saúde, verifica-se que além da oferta precoce de alguns alimentos, estes não atendem às necessidades nutricionais das crianças. A água, os chás, o leite artificial e os mingaus de cereais foram os itens da dieta com oferta iniciada antes do primeiro mês de vida.

Apesar do incentivo e valorização ao aleitamento materno, como uma das mais importantes ações de promoção e prevenção da saúde da criança, a prática adotada na comunidade pesquisada é inferior às recomendações nutricionais, o que reforça a necessidade de um trabalho consistente de incentivo e apoio à amamentação exclusiva nos primeiros seis meses de vida da criança. Os prejuízos da alimentação artificial e a introdução inadequada de alimentos devem ser discutidos com as mães fazendo-as conhecer os princípios que norteiam a alimentação infantil.

Por outro lado, espera-se que os resultados aqui apresentados sirvam para monitorar e reorientar as ações de suporte nutricional às crianças e fortaleça nas famílias atitudes e hábitos positivos para garantir alimentação infantil saudável.

\section{REFERÊNCIAS}

1. Del Ciampo LA, Saunder C. O papel da educação nutricional no combate às carências nutricionais. Rev Nutr Campinas 2000; 13 (1):11-6.

2. Ministério da Saúde. Guia alimentar para crianças menores de dois anos. Brasília; 2002.

3. Dantas Filho S, Muniz HF. Alimenta-ção da criança. In: Lima AJ. Pediatria essencial. São Paulo: Atheneu; 1998. p. 196-206.

4. Terra VM, Sarrubbo SAB. Nutrição do lactente. In: Grisi ES, Escobar A. Pediatria. São Paulo: Atheneu; 2000. p. $15-23$.

5. Akré J. Alimentação infantil: bases fisiológicas. Genebra: OMS; 1997.

6. Word Health Organization (WHO). The optimal duration of exclusive breastfeeding: a systematic review. Genebra: Department of Nutrition for Health and Development; 2002.

7. Giugliani ERJ. Aleitamento materno na pratica clinica. J Pediatr (Rio de Janeiro) 2000; 76(Supl 3):238-52.

8. Sousa FGM. Situação nutricional de ferro em crianças de seis a sessenta meses na perspectiva do Modelo Campo de Saúde [dissertação]. Fortaleza (CE): Universidade Federal do Ceará; 2002.

9. Lalonde M. A new perspective on the health of Canadians: a working document. Ottawa: Minister of National Health and Welfare; 1974.

10. Conselho Nacional de Saúde. Resolução n. 196, de 10 de outubro de 1996. Dispõe sobre as diretrizes e normas regulares de pesquisa envolvendo seres humanos. Brasília; 1996.

11. Spinelli MGN. Pratica alimentar de crianças menores de 1 ano de idade atendidas em UBS Escola do Município de Mogi das Cruzes. [dissertação]. São Paulo (SP): Faculdade de Saúde Pública da USP; 2000.

12. Ministério da Saúde. Programa Nacional de Incentivo ao Aleitamento Materno. Normas e rotinas para o incentivo ao aleitamento materno. Brasília; 1993.

13. Almeida JAG. Leite fraco: um problema da mama ou da cultura. Masto-Magazine 1998; 1(1):2.

14. Lacerda E; Cunha AJ. Anemia ferropriva e alimentação no segundo ano de vida no Rio de Janeiro. Rev Panam Salud Publica 2001; 9(5):294-301.

15. Cavalcanti MLF. Os cereais e a alimentação infantil. Temas Pediatria 1995; 1(2):3-20.

16. Ministério da Saúde. Atenção Integral às Doenças Prevalentes na Infância (AIDPI). Módulo 2. Brasília; 1999. 


\section{ANEXO A}

I- Amamentação:

\section{Instrumento para coleta de dados ${ }^{1}$}

1. Amamentou a (0)

( ) Sim

(0)

( ) Não

(nome da criança)?

2. $\mathrm{A}(0)$ (nome da criança) tomou água ou chá nos primeiros seis meses de vida?
( ) Sim
( ) Não
( ) Não sabe informar

3. ( ) Sim (nome da criança) ã̃o

4. (Se já foi desmamada). Que idade tinha quando deixou de mamar?
( ) $>$ de 1 sem
( ) $1 \mathrm{sem}$
( ) 15 dias
( ) $1 \mathrm{~m}$
( ) $3 \mathrm{~m}$
( ) $3 \mathrm{~m}$
( ) $4 \mathrm{~m}$
( ) $5 \mathrm{~m}$
( ) $7 \mathrm{~m}$
( ) $8 \mathrm{~m}$
( ) $9 \mathrm{~m}$
( ) $10 \mathrm{~m}$
( ) $14 \mathrm{~m}$
( ) $15 \mathrm{~m}$
() $17 \mathrm{~m}$
() $13 \mathrm{~m}$
( ) $19 \mathrm{~m}$
( ) $20 \mathrm{~m}$
( ) $24 \mathrm{~m}$
( ) não lembra
( ) $2 \mathrm{~m}$
( ) $11 \mathrm{~m}$
( ) $16 \mathrm{~m}$
( ) $23 \mathrm{~m}$
( ) $21 \mathrm{~m}$
( ) $22 \mathrm{~m}$
() não sabe informar

Só perguntar se na resposta anterior a criança deixou de mamar com menos de 6 meses

5. Motivos maternos alegados para a introdução de outros alimentos além do leite materno:
( ) leite fraco
( ) fissuras da mama
( ) pouco leite
( ) ingurgitamento de mama
( ) trabalho materno
( ) criança não pegou o peito
( ) criança chorava muito
( ) mama dolorida
( ) mãe faleceu
( ) mãe voltou a estudar
( ) doença/internação de outro filho
( ) mãe sentia-se fraca
( ) filho adotivo
( ) internação da mãe
( ) outros

6. Com que idade (nome da criança) começou a receber outro leite além do materno?
( ) $>$ de 1 sem
( ) $1 \mathrm{sem}$
( ) 15 dias
( ) $1 \mathrm{~m}$
( ) $2 \mathrm{~m}$
( ) $3 \mathrm{~m}$
( ) $3 \mathrm{~m}$
( ) $7 \mathrm{~m}$
( ) $8 \mathrm{~m}$
( ) $4 \mathrm{~m}$
( ) $5 \mathrm{~m}$
( ) $9 \mathrm{~m}$
( ) $12 \mathrm{~m}$
( ) $13 \mathrm{~m}$
( ) não lembra
() não sabe informar
( ) $14 \mathrm{~m}$
( ) $10 \mathrm{~m}$
( ) $6 \mathrm{~m}$
( ) $11 \mathrm{~m}$
( ) não se aplica
( ) $15 \mathrm{~m}$
( ) $16 \mathrm{~m}$

7. Com que idade você introduziu mingau na alimentação de (nome da criança)?
( ) $>$ de 1 sem
( ) 1 sem
( ) $3 \mathrm{~m}$
( ) $3 \mathrm{~m}$
( ) $7 \mathrm{~m}$
( ) $8 \mathrm{~m}$
( ) 15 dias
( ) $1 \mathrm{~m}$
( ) $2 \mathrm{~m}$
( ) $12 \mathrm{~m}$
( ) $13 \mathrm{~m}$
( ) não lembra
( ) não sabe informar
() $9 \mathrm{~m}$
( ) $14 \mathrm{~m}$
( ) $5 \mathrm{~m}$
( ) $10 \mathrm{~m}$
( ) $6 \mathrm{~m}$
( ) $15 \mathrm{~m}$
( ) $11 \mathrm{~m}$
( ) não se aplica
( ) $16 \mathrm{~m}$

8. Com que idade você introduziu sopa na alimentação de (nome da criança)?

9. Com que idade você introduziu frutas na alimentação de (nome da criança)?

10. Com que idade (nome da criança) comeu da própria comida da família?

11. Alimentos oferecidos atualmente a (nome da criança):
( ) leite materno
( ) mingaus
( ) sopas
( ) frutas amassadas
( ) comida da família
( ) outros. Quais?
( ) sucos
( ) não sabe informar
( ) não se aplica
( ) não lembra

12. Alimentos encontrados com mais freqüência na sua casa
( ) arroz
( ) feijão
( ) frango
( ) carne
( ) farinha
() ovo
( ) pão
enlatados
( ) peixe
( ) macarrão
( ) outros. Quais?

13. Quantas vezes você oferece comida para (nome da criança) nas 24 horas (de dia e de noite)? vezes.

\footnotetext{
${ }^{1}$ Baseado no Elemento Estilo de Vida do Modelo Campo de Saúde de Marc Lalond.
} 